\title{
Comprehensive circular RNA expression profile in radiation- treated HeLa cells and analysis of radioresistance-related circRNAs
}

\author{
Duo Yu ${ }^{1}$, Yunfeng Li $^{1}{ }^{\text {, }}$ Zhihui Ming ${ }^{2}$, Hongyong Wang ${ }^{1}$, Zhuo Dong ${ }^{3}$, Ling Qiu ${ }^{1}$, Tiejun Wang ${ }^{\text {Corresp. }}{ }^{1}$ \\ 1 Radiotherapy Department, 2nd Hospital Affiliated to Jilin University, Changchun, China \\ 2 Stomatology Department, 1st Hospital Affiliated to Jilin University, Changchun, China \\ ${ }^{3}$ College of Public Medicine, Key laboratory of Radiobiology, Ministry of Health, Jilin University, Changchun, China \\ Corresponding Author: Tiejun Wang \\ Email address: wangtiejunyd@sina.com
}

Background: Cervical cancer is one of the most common cancers in women worldwide. Malignant tumors develop resistance mechanisms and are less sensitive to or do not respond to irradiation.With the development of high-throughput sequencing technologies, circular RNA(circRNA) has been identified in an increasing number of diseases, especially cancers. It has been reported that circRNA can compete with microRNAs (miRNAs) to change the stabilityor translation of target RNAs, thus regulating gene expression at the transcriptional level. However, the role of circRNAs in cervical cancer and the radioresistance mechanisms of HeLa cells are unknown. The objective of this study is to investigate the role of circRNAs in radioresistance in HeLa cells. Methods: Highthroughput sequencing and bioinformatics analysis of irradiated and sham-irradiated HeLa cells. The reliability of high-throughput RNA sequencing was validated using quantitative real-time polymerase chain reaction (qRT-PCR). The most significant circRNA functions and pathways were selected by Gene Ontology(GO) and Kyoto Encyclopedia of Genes and Genomes(KEGG) analyses. A circRNA-miRNA-target gene interaction network was used to find circRNAs associated with radioresistance. Moreover, a protein-protein interaction network was constructed to identify radioresistance-related hub proteins. Results: Highthroughput sequencing allowed identifying 16,893 circRNAs involved in the response of HeLa cells to radiation. Compared with the control group, there were 153 differentially expressed circRNAs, of which 76 were up-regulated and 77 were down-regulated. GO covered three domains: biological process (BP), cellular component (CC) and molecular function (MF). The terms assigned to the BP domain were peptidyl-tyrosine dephosphorylation and regulation of cell migration. The identified CC terms were cell-cell adherens junction, nucleoplasm and cytosol, and the identified MF terms were protein binding and protein tyrosine phosphatase activity. The top five KEGG pathways were MAPK 
signaling pathway, endocytosis, axon guidance, neurotrophin signaling pathway, and SNARE interactions in vesicular transport.The PPI analysisindicated that 19 proteins might be hub proteins. Conclusions: CircRNAs may play a major role in the response to radiation. These findings may improve our understanding of the role of circRNAs in radioresistance in HeLa cells and allow the development of novel therapeutic approaches. 
1 Comprehensive circular RNA expression profile in radiation-treated

2 HeLa cells and analysis of radioresistance-related circRNAs

3 Duo Yu ${ }^{1}$, Yunfeng Li ${ }^{1}$, Zhihui Ming ${ }^{3}$, Hongyong Wang ${ }^{1}$, Zhuo Dong ${ }^{2}$, Ling Qiu $4 \quad$, Tiejun Wang ${ }^{1}$

$6 \quad{ }^{1}$ Radiotherapy Department, 2nd Hospital Affiliated toJilin University, Changchun , 7 China

$8 \quad{ }^{2}$ College of Public Medicine, Key laboratory of Radiobiology, Ministry of Health,

9 Jilin University, Changchun, China

103 Stomatology Department, $1^{\text {st }}$ Hospital Affiliated toJilin University, Changchun,

11 China

13 Corresponding Author:

14 Tiejun Wang,

15 E-mail: wangtiejunyd@sina.com

17 Abstract:

Background: Cervical cancer is one of the most common cancers in women worldwide. Malignant tumors develop resistance mechanisms and are less sensitive to or do not respond to irradiation. With the development of high-throughput

21 sequencing technologies, circular RNA(circRNA) has been identified in an 
22 increasing number of diseases, especially cancers. It has been reported that

23 circRNA can compete with microRNAs (miRNAs) to change the stabilityor

translation of target RNAs, thus regulating gene expression at the transcriptional level. However, the role of circRNAs in cervical cancer and the radioresistance mechanisms of HeLa cells are unknown. The objective of this study is to investigate the role of circRNAs in radioresistance in HeLa cells.

Methods: High-throughput sequencing and bioinformatics analysis of irradiated and sham-irradiated HeLa cells. The reliability of high-throughput RNA sequencing was validated using quantitative real-time polymerase chain reaction (qRT-PCR).The most significant circRNA functions and pathways were selected by Gene Ontology(GO) and Kyoto Encyclopedia of Genes and Genomes(KEGG) analyses. A circRNA-miRNA-target gene interaction network was used to find circRNAs associated with radioresistance. Moreover, a protein-protein interaction network was constructed to identify radioresistance-related hub proteins.

Results: High-throughput sequencing allowed identifying 16,893 circRNAs involved in the response of HeLa cells to radiation. Compared with the control group, there were 153 differentially expressed circRNAs, of which 76 were upregulated and 77 were down-regulated. GO covered three domains: biological process (BP), cellular component (CC) and molecular function (MF). The terms assigned to the BP domain were peptidyl-tyrosine dephosphorylation and regulation of cell migration. The identified CC terms were cell-cell adherens 
43 junction, nucleoplasm and cytosol, and the identified MF terms were protein

44 binding and protein tyrosine phosphatase activity. The top five KEGG pathways

45 were MAPK signaling pathway, endocytosis, axon guidance, neurotrophin

46 signaling pathway, and SNARE interactions in vesicular transport.The PPI

47 analysisindicated that 19 proteins might be hub proteins.

48 Conclusions: CircRNAs may play a major role in the response to radiation. These

49 findings may improve our understanding of the role of circRNAs in radioresistance

50 in HeLa cells and allow the development of novel therapeutic approaches.

\section{INTRODUCTION}

Cervical cancer is one of the most common cancers in women worldwide and is responsible for the high mortality of female cancers(Du XL et al., 2012). In recent years, with improvements in radiotherapy, radiochemotherapy after operation is the standard treatment for cervical cancer. However, recurrence and metastasis after radiotherapy remain a major problem in the treatment of locallyadvanced cervical cancer(de Freitas et al., 2015; Zhao et al., 2013). Therefore, it is crucial to understand the mechanisms underlying the development of

61 radioresistance to improve the therapeutic effect of radiotherapy in the treatment of 62 cervical cancer. 
64 RNA class that forms covalent closed-loop structures without 5'-3'polarity(Qu et

65 al., 2015). The development of novel biochemical and computational approaches

66 has focused RNA research on circRNAs. CircRNAs are involved in many human

67 diseases, including cancer(Burd et al., 2010b; Bachmayr-Heyda et al., 2015). Some

68 studies have demonstrated that circRNAs play a major role in the biological

69 function of a network of competing endogenous RNAs. Importantly, circRNAs

70 may compete with micro RNAs (miRNAs) to change the stability and translation

71 of target RNAs, thus regulating gene expression at the transcriptional

72 level(Bachmayr-Heyda et al., 2015; Zhong et al., 2018; Ivanov et al., 2015; Qu et

73 al., 2015). Despite this significant progress, the expression profile, mechanism of

74 action, and biological activity of circRNA have not been completely elucidated.

75 In our study, high-throughput RNA sequencing was used to investigate the

76 differential expression profiles of circRNAs between irradiated HeLa cells and

77 sham-irradiated HeLa cells. The comprehensive analysis of radioresistance-related circRNAs may lay a foundation for future studies on the diagnosis and treatment of cervical cancer.

\section{MATERIALS \& METHODS}

\section{HeLa cells and irradiation}

82 HeLa cells were purchased from Procell Life Science and Technology Co. Ltd.

83 (Wuhan, China) with short tandem repeat (STR) qualification report

84 (Supplementary data). The cells were cultured in MEM medium (Gibco, Life 
85 Technologies Inc., Grand Island, NY, USA)supplemented with 10\% fetal bovine

86

87

serum (Sigma), 1\% antibiotic-antimitotic solution (Gibco, Life Technologies).An

X-ray generator (Model X-RAD320i X; Precision X-ray, Inc., North Branford, USA) was used to deliver radiation at a dose rate of $1.020 \mathrm{~Gy} / \mathrm{min}(180 \mathrm{kV} ; 20$ $\mathrm{mA}$ ) for a total dose of $10 \mathrm{~Gy}$ in the treatment group(irradiated). The control group (sham-irradiated) did not receive radiation as blank comparison. Each group had three samples.

\section{High-throughput sequencing of circRNAs and differential expression analysis}

Total RNA was extracted from each group by using TRIzol reagent (Invitrogen, Carlsbad, USA) following the manufacturer's protocol. RNA quantity and quality were determined in a Nano Drop ND-1000 spectrophotometer (Nano Drop Technologies, Inc., Wilmington, USA). RNA integrity was evaluated by standard denaturing agarose gel electrophoresis using the Agilent 2100 Bioanalyzer. The total RNA was measured in a spectrophotometer at the wavelengths of $260 \mathrm{~nm}$ and $280 \mathrm{~nm}$. The samples with an OD 260/280 ratio of approximately 2.0 were used. CircRNAs were quantitatively analyzed by Novogene Bioinformatics Technology Co.Ltd (Beijing, China). After removal of ribosomal RNA and building a library, high-throughput RNA sequencing was performed. Find_circ was used to identify circRNAs(Memczak et al., 2013). Differentially expressed circRNAs were detected by the negative binomial distribution test using the DESeq2 package. Two criteria were chosen: (i) $\mid \log 2$ (foldchange) $\mid>1$ and (ii) $P$-value $(P<0.05)$. 
106

107

108

109

110

111

112

113

114

115

116

117

118

119

120

121

122

123

124

125

126

\section{Construction of the circRNA-miRNA-target gene interaction network}

Mounting evidence has indicated that circRNAs may regulate the activity of

miRNAs by acting as competing endogenous RNAs or miRNA sponges. The role of circRNAs in HeLa cells subjected to radiation was determined by building a circRNA-miRNA-target gene co-expression network. We used the top four downregulated and top four up-regulated circRNAs to construct this network using Cytoscape software version 3.2.1. Putative interactions between miRNAs and circRNAs were evaluated using miRanda(3.3a). The top five (highest targeting relationship score) miRNAs were selected, and the target genes of these miRNAs were predicted using miRDB(http://www.mirdb.org). Target scores $>98$ were selected to construct the circRNA-miRNA-mRNA interaction network.

\section{GO and KEGG pathway analysis}

The Gene Ontology (GO) analysis provides a controlled terminology to describe gene and gene product attributes for many organisms. In this study, DAVID (https://david.ncifcrf.gov/) was used to analyze the potential function of target genes. P-values $<0.05$ were considered statistically significant. GO covers three domains: biological process(BP), cellular component(CC), and molecular function(MF). The five most enriched GO terms were ranked by p-value. KEGG pathway analysis was conducted to determine the involvement of target genes in different biological pathways. DAVID (https://david.ncifcrf.gov/) was also used in this part of the analysis. 
127 Protein-protein interaction(PPI) network analysis

128 The online tool STRING (https://string-db.org) was used to analyze the target

genes. The selection criterion was the highest confidence (interaction score $>0.9$ ).

130 Cytoscape software version 3.2.1 was used to draw the PPI network. The hub

131 proteins were selected by their association with other proteins. The target genes

132 with more associations have important roles in the PPI interaction network.

133 Analysis of the expression level of circRNAs using qRT-PCR

134 The reliability of high-throughput RNA sequencing was validated. The top four up-regulated circRNAs and top four down-regulated circRNAs were selected. qRT-PCR was performed using Q SYBR green Supermix (Bio Rad), and PCRspecific amplification was conducted in the 7900 HT Sequence Detection System (ABI PRISM, USA). The expression was determined by using the threshold cycle $(\mathrm{Ct})$, and relative expression levels were calculated via the $2^{-\triangle \Delta \mathrm{Ct}}$ method. GAPDH served as the standard internal control and all reactions were performed in triplicate.

Results

Expression pattern of circRNAs during the priming phase of radiation-treated

HeLa cells

High-throughput sequencing was used to determine the expression profile of circRNAs. Two groups of HeLa cells were used, and each group contained three samples. The correlation between gene expression levels in different samples 
148 indicated the biological repetition (Figure 1A). The analysis of sequencing data 149 allowed identifying 16,893 circRNAs in HeLa cells. The length of HeLa circRNA 150 candidates ranged from $<150$ to 99,934 nucleotides (nt). Approximately $67 \%$ of 151 circRNAs had the predicted spliced length of $<10,000 \mathrm{nt}$, whereas $46.4 \%$ and $32.0 \%$ 152 of the circRNAs had a length of $<5,000$ and 10,000-50,000 nt, respectively (Figure 153 1B). Among the 16,893 circRNAs, 11,456 were detected in the control group, 11,018 were detected in the treatment group, and 5,581 were detected in both groups. The hierarchical clustering showed the gene expression patterns of the samples (Figures $1 \mathrm{C}$ and D.).

157

158

159

160

161

162

163

164

165

166

167 168

\section{Identification of differentially-expressed circRNAs in Hela cells}

The $\log _{2}$ (foldchange) $\mid>1$ and $\mathrm{p}$-value $(\mathrm{p}<0.05)$ were used to evaluate significant differences in the expressions of circRNAs between the two groups. A total of 153 circRNAs were differentially expressed in the Treatment Group compared with the Control Group. Of these, the volcano plot indicated that 76 circRNAs were upregulated and 77 were down-regulated (Figure 1E). The novel genomic feature of circRNAs in both groups is shown in Figure 1F.

\section{Validation of circRNA expression}

To verify the high-throughput sequencing results, the top four up-regulated and top four down-regulated circRNAs were selected and their expression levels were validated by qRT-PCR analysis. The qRT-PCR results are shown in Figure 2. hsa_circ_0009035, hsa_circ_0000392, hg38_circ_0004913, hsa_circ_0004015 
169

170

171

172

173

174

175

176

177

178

179

180

181

182

183

184

185

186

187

188

189

were significantly up-regulated in the Treatment Group compared with the Control Group, whereas hg38_circ_0013682, hg38_circ_0015954, hsa_circ_0013738, and hsa_circ_0013225 were down-regulated in the Treatment Group. The expression patterns of these eight circRNAs determined by qRT-PCR agreed with the results of the high-throughput sequencing analysis. The sequence of the primers used in qRT-PCR is shown in Table 1.

\section{Construction of the circRNA-miRNA-target gene interaction network}

A circRNA-miRNA-target gene co-expression network was constructed to evaluate the potential functions of circRNAs. Cytoscape was used to draw the circRNA-miRNA-target gene interaction network (Figure 3). It is of interest that circRNAs may play a central role in this interaction network, and a single circRNA may correlate with many miRNAs and regulate many target genes. This network may help elucidate the potential function of circRNAs and their mechanism of action.

\section{GO and KEGG analysis of target genes}

The role of circRNAs in response to radiation was investigated by performinga GO functional analysis. The target genes from the circRNA-miRNA-target gene interaction network were used in this analysis. GO has three different domains:biological process (BP), cellular component (CC), and molecular function (MF). The top five(ranked by p-value) BP terms, CC terms, and MF terms are shown in Figure 4 and Table 2. The identified BP terms were peptidyl-tyrosine 
190

191

192

193

194

195

196

197

198

199

200

201

202

203

204

205

206

207

208

209

210

dephosphorylation, regulation of cell migration, positive regulation of transcription from RNA polymerase II promoter, angiogenesis, and morphogenesis of an epithelial sheet. The identified CC terms were cell-cell adherens junction, nucleoplasm, cytosol, lamellipodium, and nucleus. The identified MF terms were protein binding, tyrosine phosphatase activity, cadherin binding involved in cellcell adhesion, RNA polymerase II core promoter proximal region sequencespecific DNA binding, transcriptional activator activity, and RNA polymerase II core promoter proximal region sequence-specific binding. The top five(ranked by gene count) KEGG pathways are shown in Figure 5and Table 3. The identified pathways were MAPK signaling, endocytosis, axon guidance, neurotrophin signaling, and SNARE interactions in vesicular transport. It is of note that five genes-RPS6KA5, RPS6KA6, CRKL, RAP1A, FASLG, MAPK8-were found in both the MAPK and neurotrophin signaling pathway.

\section{PPI network}

STRING was used to predict protein interactions between the target genes. Interaction scores higher than 0.9 (highest confidence) were selected for constructing PPI networks (Figure 6). Nineteen proteins-IGF2R, DNAJC6, FZD4, CBL, CLTC, ARPC3, VAMP2, ITGB3, VAMP4, ITGB1, MAPK8, KIF2A, CLASP1, SMC3, STAG2, STAG1, H2AFZ, RBBP4, and UBE2D3-were strongly correlated with other proteins (connected with $>10$ proteins). These hub proteins might play a crucial role in the radioresistance of cervical cancer. 
211

212

213

214

215

216

217

218

219

220

221

222

223

224

225

226

227

228

229

230

231

\section{DISCUSSION}

The development of high-throughput sequencing technology has increased our understanding of the role of circRNAs. A recent study found that circRNAs are important members of the non-coding RNA family in different species. The role of circRNA in human disease, especially in cancer, is vital. The functions and biological characteristics of circRNAs may help elucidate the mechanisms underlying human disease. Radioresistance is considered a severe complication of cervical cancer radiotherapy, and the radioresistance mechanism remains poorly understood. In this study, circRNA sequencing was used to analyze the circRNA expression profile between irradiated HeLa cells and sham-irradiated HeLa cells and explore the possible involvement of differentially-expressed circRNAs in radioresistance mechanisms.

In the present study, a 10Gy X-ray was applied to HeLa cells, which were collected $48 \mathrm{~h}$ later. This interval was chosen because most highly-expressed radiation resistance-related genes are strongly inhibited by radiation after $48 \mathrm{~h}$ (Shen et al., 2017b; Ghosh \& Krishna, 2012b; Balkwill, 2004; Zhang et al., 2017). Highthroughput RNA sequencing allowed identifying 16,893 circRNAs in both Treatment and Control Group. A total of 153 circRNAs was differentially expressed $(\mathrm{p}<0.05, \mid \log 2($ foldchange $) \mid>1)$. Four up-regulated and four downregulated circRNAs validated by qRT-PCR were significantly dysregulated, and this result is consistent with the high-throughput sequencing, indicating the high 
232 reliability of high-throughput sequencing data.

233 A circRNA-miRNA-target gene interaction network was constructed to

234 further investigate the regulatory role of circRNAs in radioresistance. This

235 network showed that circRNAs might play a central regulatory role. A single

236 circRNA may be associated with many miRNAs and then may regulate more

237 target genes. For instance, in our study, hsa_circ_0004015 may bind to hsa-miR-

238

3163, hsa-miR-3065-5p, hsa-miR-551b-5p, hsa-miR-4311, and hsa-miR-875-3p.

239

In turn, these miRNAs may be associated with 380 target genes, including $Z Y X$,

240

PRKAR1A, BTAF1, LRP3, andETS1.

241

The potential regulatory role of circRNAs in radioresistance was further

242

investigated by conducting KEGG and GO analyses and determining the

243

differential expression of mRNAs in the two study groups. GO analysis was

244

performed to annotate the BPs, CCs, and MFs of target genes. The GO analysis

245

results indicated that the high expression of target genes might be involved in cell

246

migration and angiogenesis. Many studies have shown that radiation can kill

247

tumor cells and promote cell migration and invasion via different mechanisms. It

248

was shown that sublethal X-ray can enhance cell migration(Imaizumi et al., 2018).

249

Another study found that the higher was radioresistance, the higher was the

250

expression of $V E G F R$, which might lead to fast angiogenesis(Lee et al., 2017).

251

"Protein binding" was an important MF-related GO term associated with radiation.

252

As for protein binding, it was reported that the specific binding of $V D R$ and $p 53$ in 
253

254

255

256

257

258

259

260

261

262

263

264

265

266

267

268

269

270

271

272

273

irradiated HEK 293T was primarily related to cell fate decision(Pemsel et al., 2018).

Moreover, KEGG analysis indicated that the MAPK signaling pathway was the most enriched pathway, with 19 related genes. This classic pathway is highly conserved and is involved in many cellular functions, including cell proliferation, differentiation, and migration, which is consistent with the results of GO analysis.

A study showed that baseline MAPK signaling activity conferred intrinsic radioresistance to KRAS-mutant colorectal carcinoma cells by rapid up-regulation of $h n R N P K($ Eder et al., 2017). Moreover, quiescent G0 cells were found to be more resistant to ionizing radiation than $\mathrm{G} 1$ cells because $P 38 M A P K$, phosphorylated $P 38 M A P K$, and $R A C 2$ were regulated in mutual feedback and negative feedback regulatory pathways, leading to the radioresistance of G0 cells(Pei et al., 2017). Interestingly, RPS6KA5, RPS6KA6, CRKL, RAP1A, FASLG, and MAPK8 were found in both MAPK and neurotrophin signaling pathway.

These genes may be involved in the crosstalk between different signaling pathways, but this hypothesis needs to be tested in further study.

Furthermore, in the PPI network analysis, 19 proteins were shown to associated with $>10$ other proteins and thus maybe considered as hub proteins in this network. Protein MAPK8 was associated with 10 other proteins and was also found in the MAPK and neurotrophin signaling pathwayin KEGG analysis. Studies on this gene encoding MAPK8 suggest that this kinase plays a key role in 
274

275

276

277

278

279

280

281

282

283

284

285

286

287

288

289

290

291

292

293

294

295

296

297

298

$\mathrm{T}$ cell proliferation, apoptosis, and differentiation, and may be a crucial protein in cervical cancer radioresistance.

In summary, this study determined the profile of differentially expressed circRNAs and their target genes in HeLa cells subjected to radiation. Our results suggest that circRNAs may play a major role in the response to radiation.

Considering that only a small percentage of the total circRNA population has been studied to date, the investigation of circRNAs is only beginning. Therefore, additional studies are needed to clarify the importance of circRNAs in the response to radiation and may help elucidate the biological and molecular mechanisms underlying radioresistance in HeLa cells and allow the development of novel therapeutic approaches. The exploration of radioresistance-related circRNAs in radiation-treated HeLa cells may help provide responsive therapy in therapyresistant cancer and improve prognosis.

\section{Acknowledgements:}

We thank Professor Jin Shunzi for guiding us about the bioinformatics.

\section{References:}

Balkwill, F. 2004. Cancer and the chemokine network. NATURE REVIEWS CANCER 4:540-550. 10.1038/nrc1388

Burd, C.E., Jeck, W.R., Liu, Y., Sanoff, H.K., Wang, Z., and Sharpless, N.E. 2010b. Expression of linear and novel circular forms of an INK4/ARF-associated non-coding RNA correlates with atherosclerosis risk. PLoS Genetics 6:e1001233. 10.1371/journal.pgen.1001233

de Freitas, A.C., Gomes, L.M.C., and Coimbra, E.C. 2015. Prospects of molecularly-targeted therapies for cervical cancer treatment. CURRENT DRUG TARGETS 16:77-91.

Du XL, Tao, J., Sheng, X.G., Lu, C.H., Yu, H., Wang, C., Song, Q.Q., Li, Q.S., and Pan, C.X. 2012. Intensity-
} 
modulated radiation therapy for advanced cervical cancer: a comparison of dosimetric and clinical outcomes with conventional radiotherapy. GYNECOLOGIC ONCOLOGY 125:151-157. 10.1016/j.ygyno.2011.12.432

Eder, S., Arndt, A., Lamkowski, A., Daskalaki, W., Rump, A., Priller, M., Genze, F., Wardelmann, E., Port, M., and Steinestel, K. 2017. Baseline MAPK signaling activity confers intrinsic radioresistance to KRAS-mutant colorectal carcinoma cells by rapid upregulation of heterogeneous nuclear ribonucleoprotein $\mathrm{K}$ ( $\mathrm{hnRNP} \mathrm{K}$ ). CANCER LETTERS 385:160-167. 10.1016/j.canlet.2016.10.027

Ghosh, S., and Krishna, M. 2012b. Role of Rad52 in fractionated irradiation induced signaling in A549 lung adenocarcinoma cells. Mutat Res 729:61-72. 10.1016/j.mrfmmm.2011.09.007

Imaizumi, H., Sato, K., Nishihara, A., Minami, K., Koizumi, M., Matsuura, N., and Hieda, M. 2018. X-rayenhanced cancer cell migration requires the linker of nucleoskeleton and cytoskeleton complex. CANCER SCIENCE 109:1158-1165. 10.1111/cas.13545

Ivanov, A., Memczak, S., Wyler, E., Torti, F., Porath, H.T., Orejuela, M.R., Piechotta, M., Levanon, E.Y., Landthaler, M., Dieterich, C., and Rajewsky, N. 2015. Analysis of intron sequences reveals hallmarks of circular RNA biogenesis in animals. Cell Reports 10:170-177. 10.1016/j.celrep.2014.12.019

Lee, C., Shim, S., Jang, H., Myung, H., Lee, J., Bae, C.H., Myung, J.K., Kim, M.J., Lee, S.B., Jang, W.S., Lee, S.J., Kim, H.Y., Lee, S.S., and Park, S. 2017. Human umbilical cord blood-derived mesenchymal stromal cells and small intestinal submucosa hydrogel composite promotes combined radiation-wound healing of mice. CYTOTHERAPY 19:1048-1059. 10.1016/j.jcyt.2017.06.007

Memczak, S., Jens, M., Elefsinioti, A., Torti, F., Krueger, J., Rybak, A., Maier, L., Mackowiak, S.D., Gregersen, L.H., Munschauer, M., Loewer, A., Ziebold, U., Landthaler, M., Kocks, C., le Noble, F., and Rajewsky, N. 2013. Circular RNAs are a large class of animal RNAs with regulatory potency. NATURE 495:333-338. 10.1038 /nature11928

Pei, H., Zhang, J., Nie, J., Ding, N., Hu, W., Hua, J., Hirayama, R., Furusawa, Y., Liu, C., Li, B., Hei, T.K., and Zhou, G. 2017. RAC2-P38 MAPK-dependent NADPH oxidase activity is associated with the resistance of quiescent cells to ionizing radiation. CELL CYCLE 16:113-122. 10.1080/15384101.2016.1259039

Pemsel, A., Rumpf, S., Roemer, K., Heyne, K., Vogt, T., and Reichrath, J. 2018. Tandem Affinity Purification and Nano HPLC-ESI-MS/MS Reveal Binding of Vitamin D Receptor to p53 and other New Interaction Partners in HEK 293T Cells. ANTICANCER RESEARCH 38:1209-1216. 10.21873/anticanres.12341

Shen, L.F., Zhao, X., Zhou, S.H., Lu, Z.J., Zhao, K., Fan, J., and Zhou, M.L. 2017b. In vivo evaluation of the effects of simultaneous inhibition of GLUT-1 and HIF-1alpha by antisense oligodeoxynucleotides on the radiosensitivity of laryngeal carcinoma using micro 18F-FDG PET/CT. Oncotarget 8:34709-34726. 10.18632/oncotarget.16671

Zhang, H., Yue, J., Jiang, Z., Zhou, R., Xie, R., Xu, Y., and Wu, S. 2017. CAF-secreted CXCL1 conferred radioresistance by regulating DNA damage response in a ROS-dependent manner in esophageal squamous cell carcinoma. Cell Death \& Disease 8:e2790. 10.1038/cddis.2017.180

Zhao, S., Yao, D.S., Chen, J.Y., and Ding, N. 2013. Aberrant expression of miR-20a and miR-203 in cervical cancer. Asian Pac J Cancer Prev 14:2289-2293.

Zhong, Y., Du Y, Yang, X., Mo, Y., Fan, C., Xiong, F., Ren, D., Ye, X., Li, C., Wang, Y., Wei, F., Guo, C., Wu, X., Li, X., Li, Y., Li, G., Zeng, Z., and Xiong, W. 2018. Circular RNAs function as ceRNAs to regulate and control human cancer progression. Molecular Cancer 17:79. 10.1186/s12943-018-0827-8

\section{Figure legends}


339

340

341

342

343

344

345

346

347

348

349

350

351

352

353

354

355

356

357

358

359

Figure1. Basic situation of high-throughput sequencing A. Inter-sample expression correlation test. Two groups of HeLa cells were used and each group contained three samples. The cell groups were treated by different methods. The correlation between gene expression levels in different samples indicated the biological repetition. The greater the absolute value of $r$ indicates that the stronger the correlation. B. CircRNA length distribution. Approximately $67 \%$ of circRNAs had the predicted spliced length of $<10,000 \mathrm{nt}$, whereas $46.4 \%$ and $32.0 \%$ of the circRNAs had a length of $<5,000$ and $10,000-50,000 \mathrm{nt}$, respectively. C. Venn diagram of the differential expression of circRNAs. The yellow part of the circle is the Control group and the purple part of the circle is the Treatment group.The total number of the circRNAs was 16,893.Approximately 11,456 circRNAs were detected in the control group, 11,018 in the treatment group, and 5,581 in both groups.D. Hclusterheatmap.Hierarchical clustering showing the differential expression profile of circRNAs between the two study groups and the homogeneity within each group.E.Volcano plot of differentially expressed circRNAs. The green and red dots in the plot represent the differentially expressed circRNAs with statistical significance. The red dots correspond to upregulated circRNAs and the green dots correspond to downregulated circRNAs.F. circRNA genomic feature data. It shows the novel genomic feature of circRNAs in both groups. Red part is exon, green part is intergenic, blue part is intron.

Figure 2.qRT-PCR results. The expression patterns of the top four up-regulated 
360

361

362

363

364

365

366

367

368

369

370

371

372

373

374

375

376

377

378

379

380

and top four down-regulated circRNAs as monitored by qRT-PCR. The expression patterns of these eight circRNAs determined byqRT-PCR agreed with the results of the high-throughput sequencing analysis and demonstrated the reliability of the RNA high-throughput sequencing technology.

Figure 3.CircRNA-miRNA-target gene interaction network. The top four down- and up-regulated circRNAs were used to construct this network using Cytoscape. Putative interactions between miRNAs and circRNAs were evaluated using miRanda. The top five (highest targeting relationship score) miRNAs were selected, then the same miRNAs' target genes were predicted using miRDB. Target scores $>98$ were selected.In this figure, rectangles represent mRNAs while the ovals represent circRNAs and triangles represent miRNAs.

Figure 4.Top five GO terms from BP, CC and MF.The top five GO terms in each group were ranked by p-value. They are peptidyl-tyrosine dephosphorylation; regulation of cell migration; positive regulation of transcription from RNA polymerase II promoter; angiogenesis; morphogenesis of an epithelial sheet(from BP). cell-cell adherens junction; nucleoplasm; cytosol; lamellipodium; nucleus(from CC). protein binding; protein tyrosine phosphatase activity; cadherin binding involved in cell-cell adhesion; RNA polymerase II core promoter proximal region sequence-specific DNA binding; transcriptional activator activity, RNA polymerase II core promoter proximal region sequence-specific binding(from MF). 
381 Figure 5.KEGG analysis. KEGG pathway analysis was conducted to determine 382 the involvement of target genes in different biological pathways. The size of each 383 circle indicates the number of circRNAs. The color of the circle indicates the p384 value. The larger of the circle and the lower of the p-value, the enriched and 385 meaningful of the pathway.

386 Figure 6.PPI network. STRING was used to predict protein interactions among 387 the target genes. The interaction scores $>0.9$ (highest confidence) were selected for 388 constructing PPI networks. The ovals represent proteins with $>10$ relationships with 389 other proteins and may be the hub proteins in this network. 390 


\section{Figure 1}

Basic situation of high-throughput sequencing

A. Inter-sample expression correlation test. Two groups of HeLa cells were used and each group contained three samples. The cell groups were treated by different methods. The correlation between gene expression levels in different samples indicated the biological repetition. The greater the absolute value of $r$ indicates that the stronger the correlation. $B$. CircRNA length distribution. Approximately $67 \%$ of circRNAs had the predicted spliced length of $<10,000 \mathrm{nt}$, whereas $46.4 \%$ and $32.0 \%$ of the circRNAs had a length of $<5,000$ and 10,000-50,000 nt, respectively. C. Venn diagram of the differential expression of circRNAs. The yellow part of the circle is the Control group and the purple part of the circle is the Treatment group. The total number of the circRNAs was 16,893.Approximately 11,456 circRNAs were detected in the control group, 11,018 in the treatment group, and 5,581 in both groups. D. Hclusterheatmap. Hierarchical clustering showing the differential expression profile of circRNAs between the two study groups and the homogeneity within each group. $\mathrm{E}$. Volcano plot of differentially expressed circRNAs. The green and red dots in the plot represent the differentially expressed circRNAs with statistical significance. The red dots correspond to upregulated circRNAs and the green dots correspond to downregulated circRNAs.F. circRNA genomic feature data. It shows the novel genomic feature of circRNAs in both groups. Red part is exon, green part is intergenic, blue part is intron. 


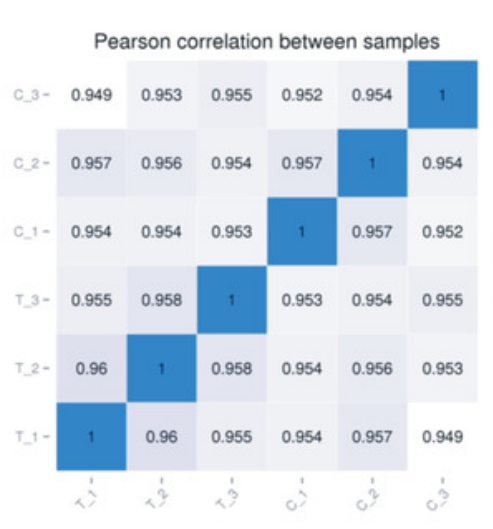

A

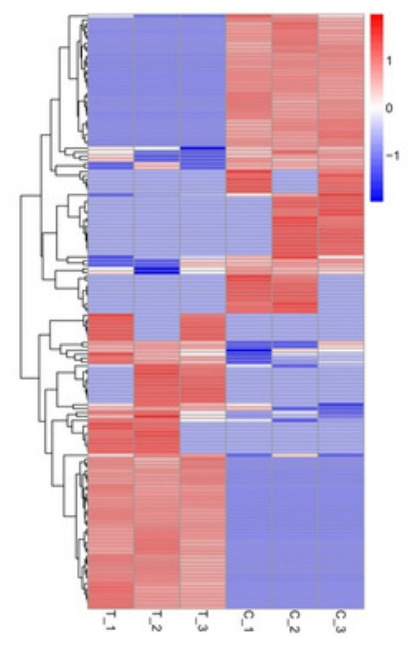

D

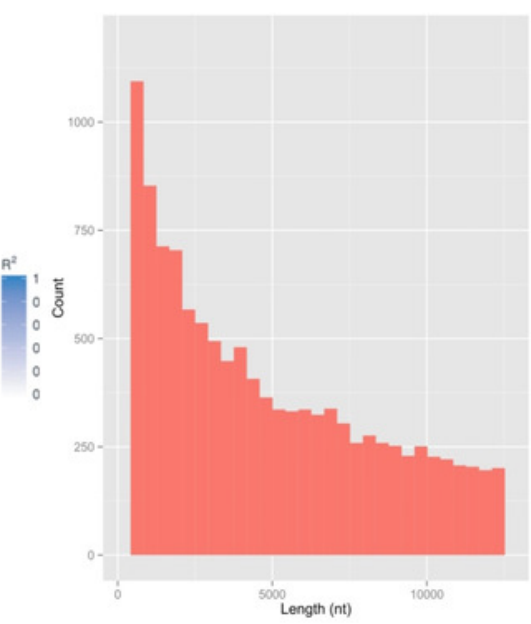

B

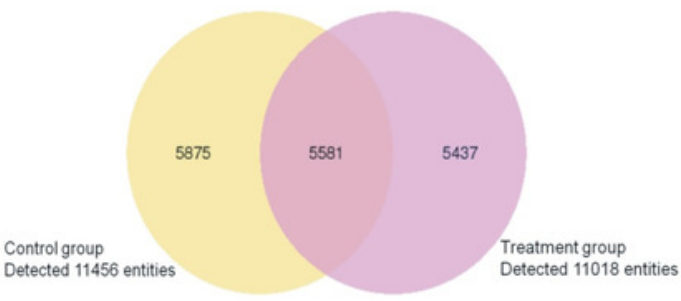

C

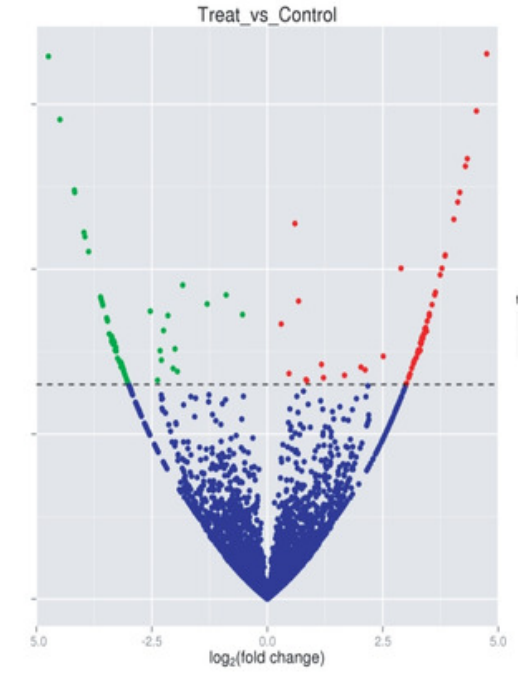

E

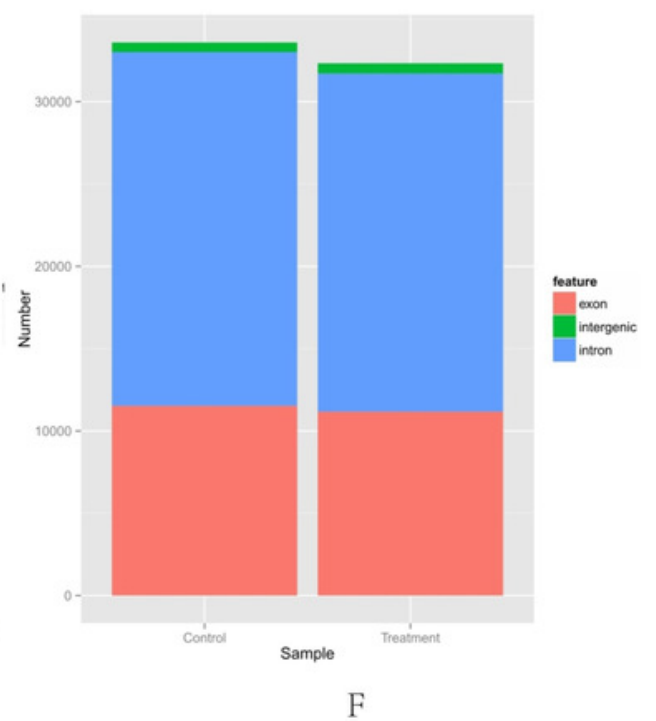


Figure 2

qRT-PCR results

The expression patterns of the top four up-regulated and top four down-regulated circRNAs as monitored by qRT-PCR. The expression patterns of these eight circRNAs determined by qRT-PCR agreed with the results of the high-throughput sequencing analysis and demonstrated the reliability of the RNA high-throughput sequencing technology.
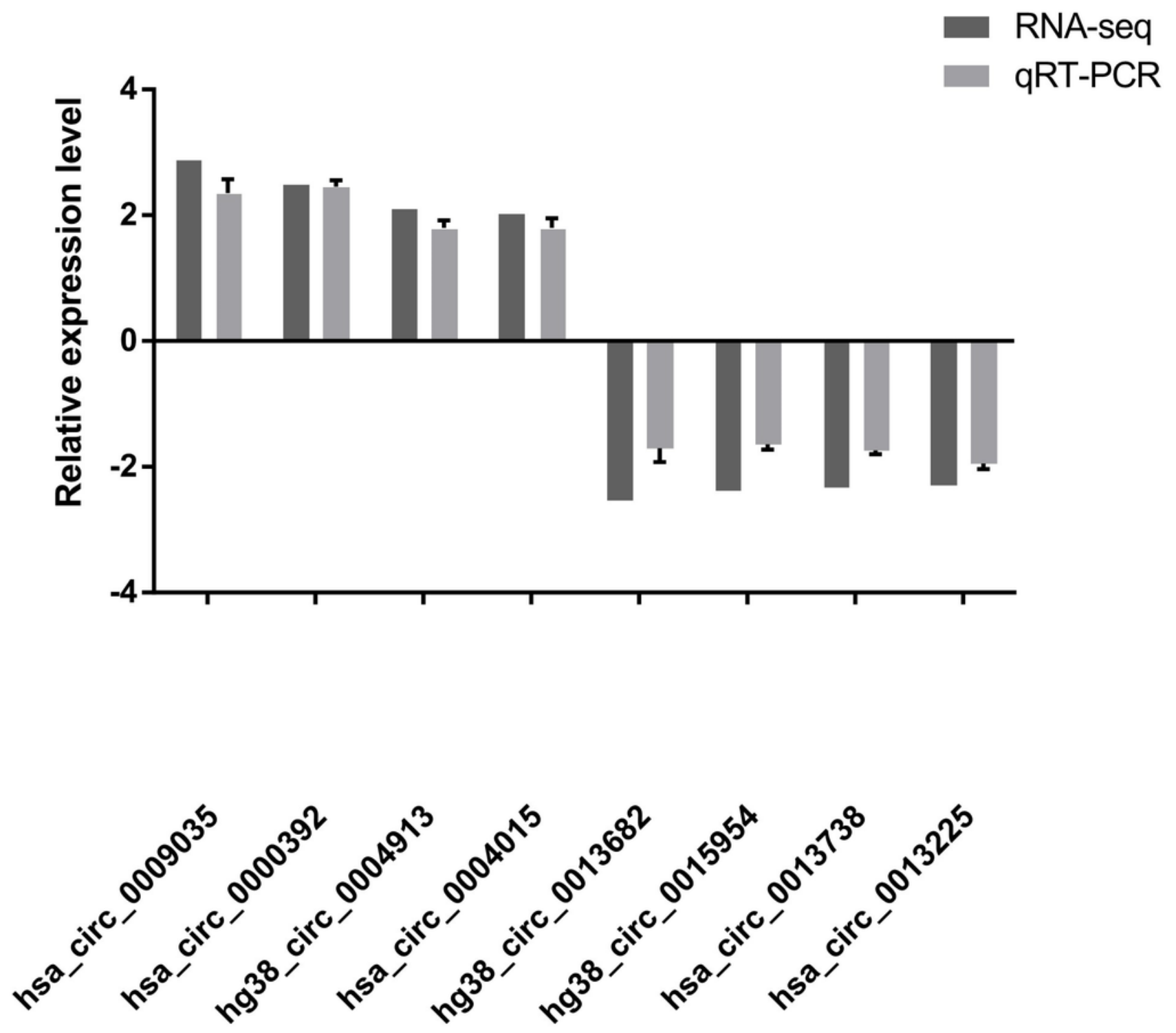


\section{Figure 3}

CircRNA-miRNA-target gene interaction network

The top four down- and up-regulated circRNAs were used to construct this network using Cytoscape. Putative interactions between miRNAs and circRNAs were evaluated using miRanda. The top five (highest targeting relationship score) miRNAs were selected, then the same miRNAs' target genes were predicted using miRDB. Target scores $>98$ were selected.In this figure, rectangles represent mRNAs while the ovals represent circRNAs and triangles represent miRNAs. 


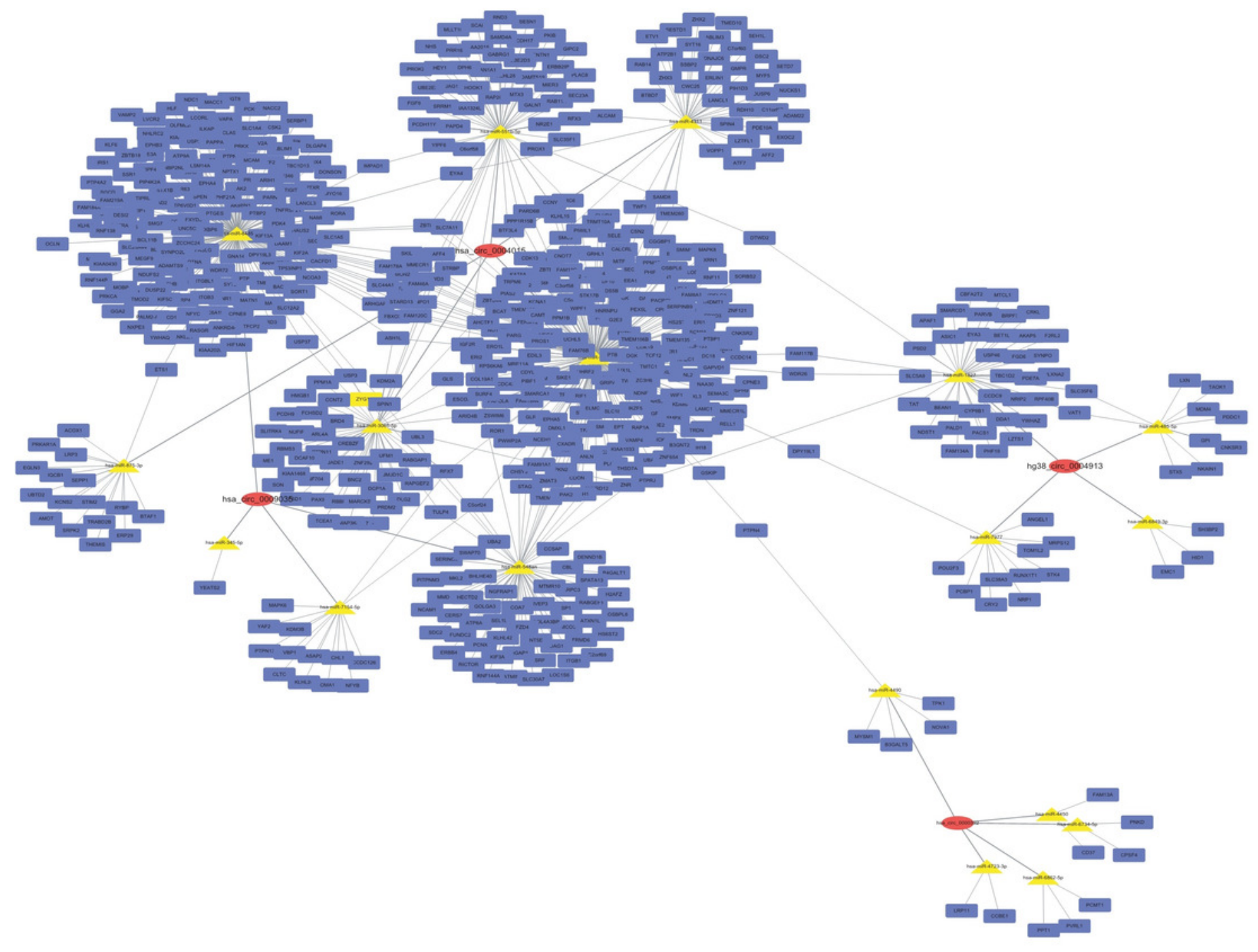




\section{Figure 4}

Top five GO terms from BP, CC and MF

The top five $\mathrm{GO}$ terms in each group were ranked by $\mathrm{p}$-value. They are peptidyl-tyrosine dephosphorylation; regulation of cell migration; positive regulation of transcription from RNA polymerase II promoter; angiogenesis; morphogenesis of an epithelial sheet (from BP).

cell-cell adherens junction; nucleoplasm; cytosol; lamellipodium; nucleus (from CC). protein binding; protein tyrosine phosphatase activity; cadherin binding involved in cell-cell adhesion; RNA polymerase II core promoter proximal region sequence-specific DNA binding; transcriptional activator activity, RNA polymerase II core promoter proximal region sequencespecific binding (from MF).

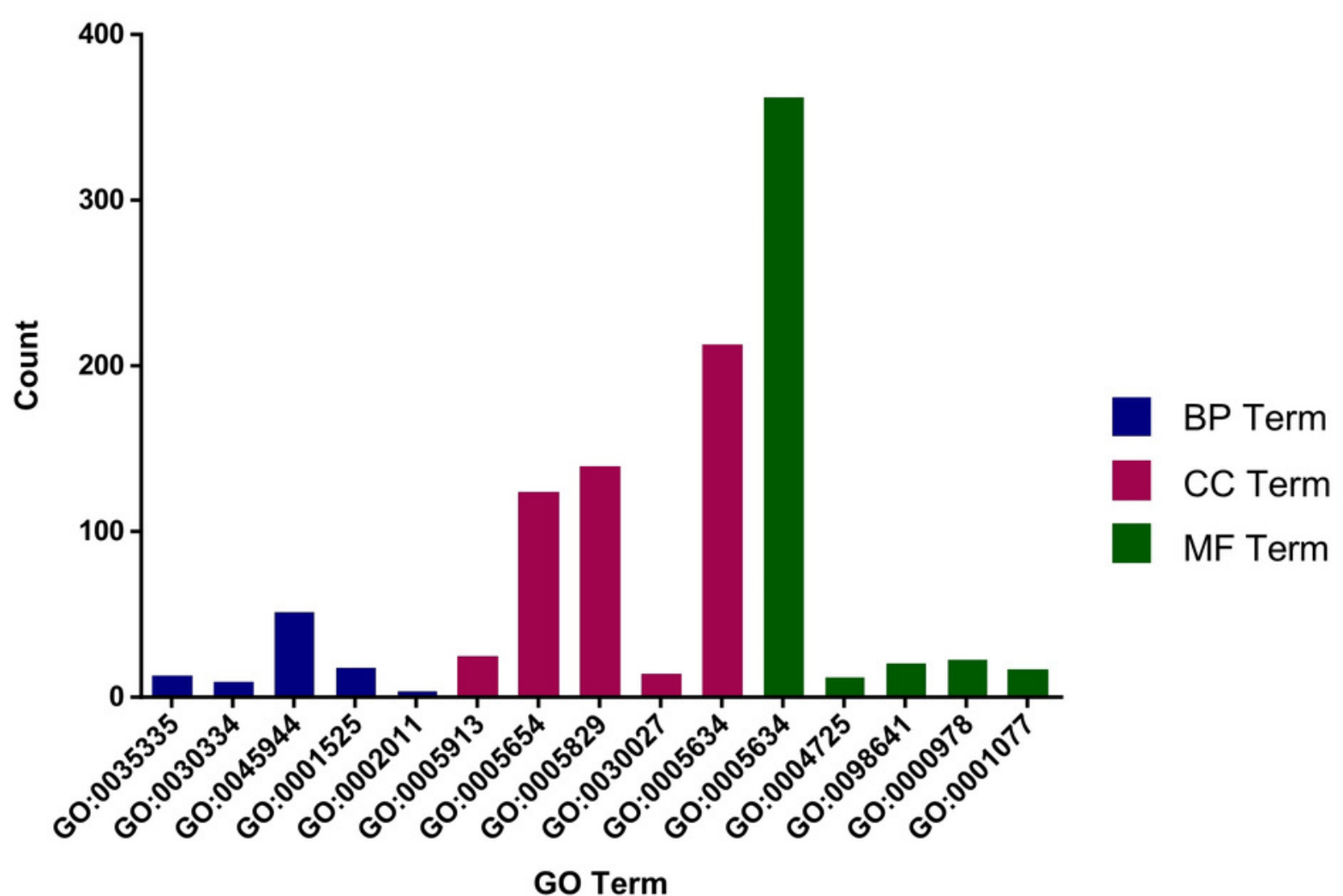




\section{Figure 5}

\section{KEGG analysis}

KEGG pathway analysis was conducted to determine the involvement of target genes in different biological pathways. The size of each circle indicates the number of circRNAs. The color of the circle indicates the p-value. The larger of the circle and the lower of the p-value, the enriched and meaningful of the pathway. 


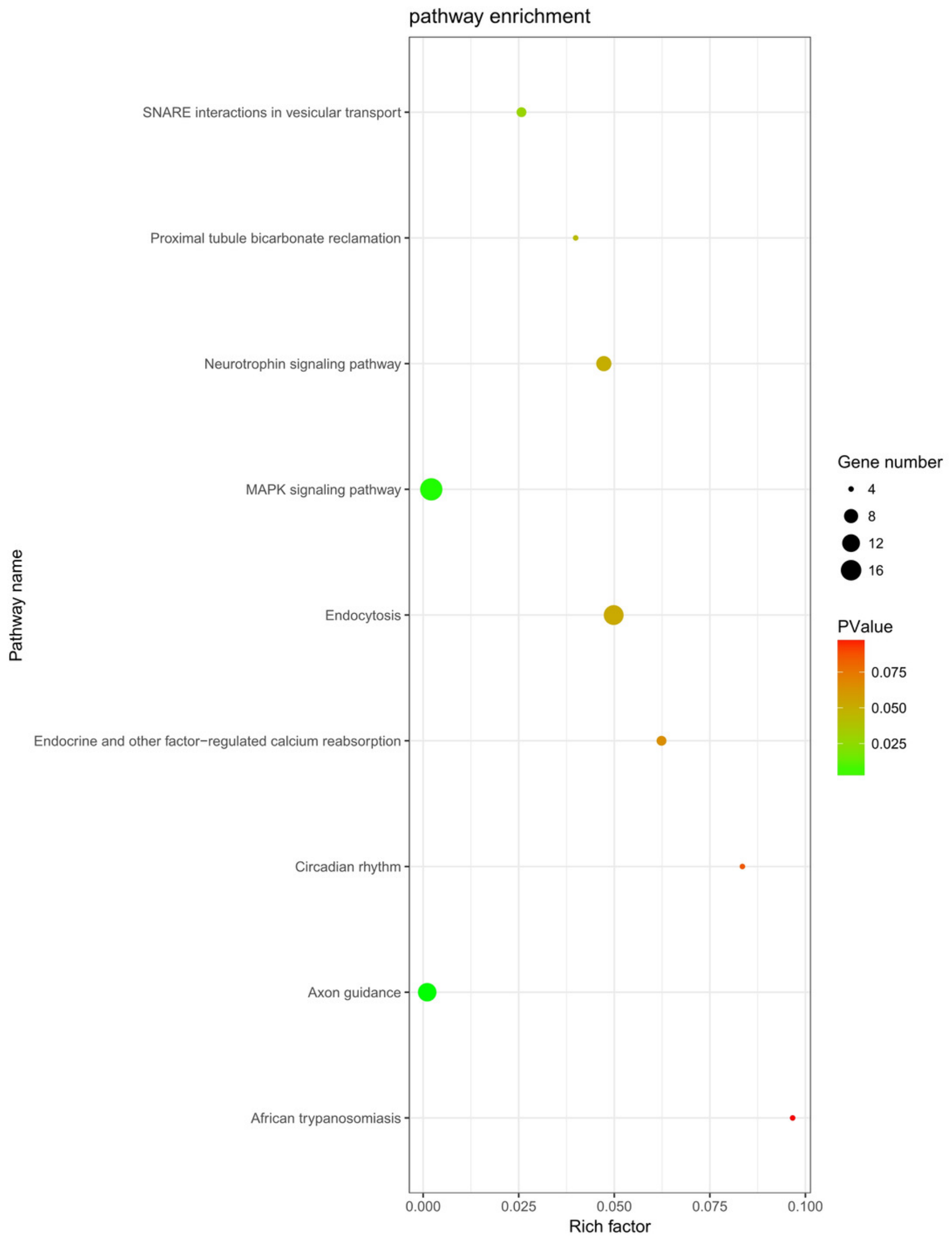




\section{Figure 6}

PPI network

STRING was used to predict protein interactions among the target genes. The interaction scores $>0.9$ (highest confidence) were selected for constructing PPI networks. The ovals represent proteins with $>10$ relationships with other proteins and may be the hub proteins in this network.

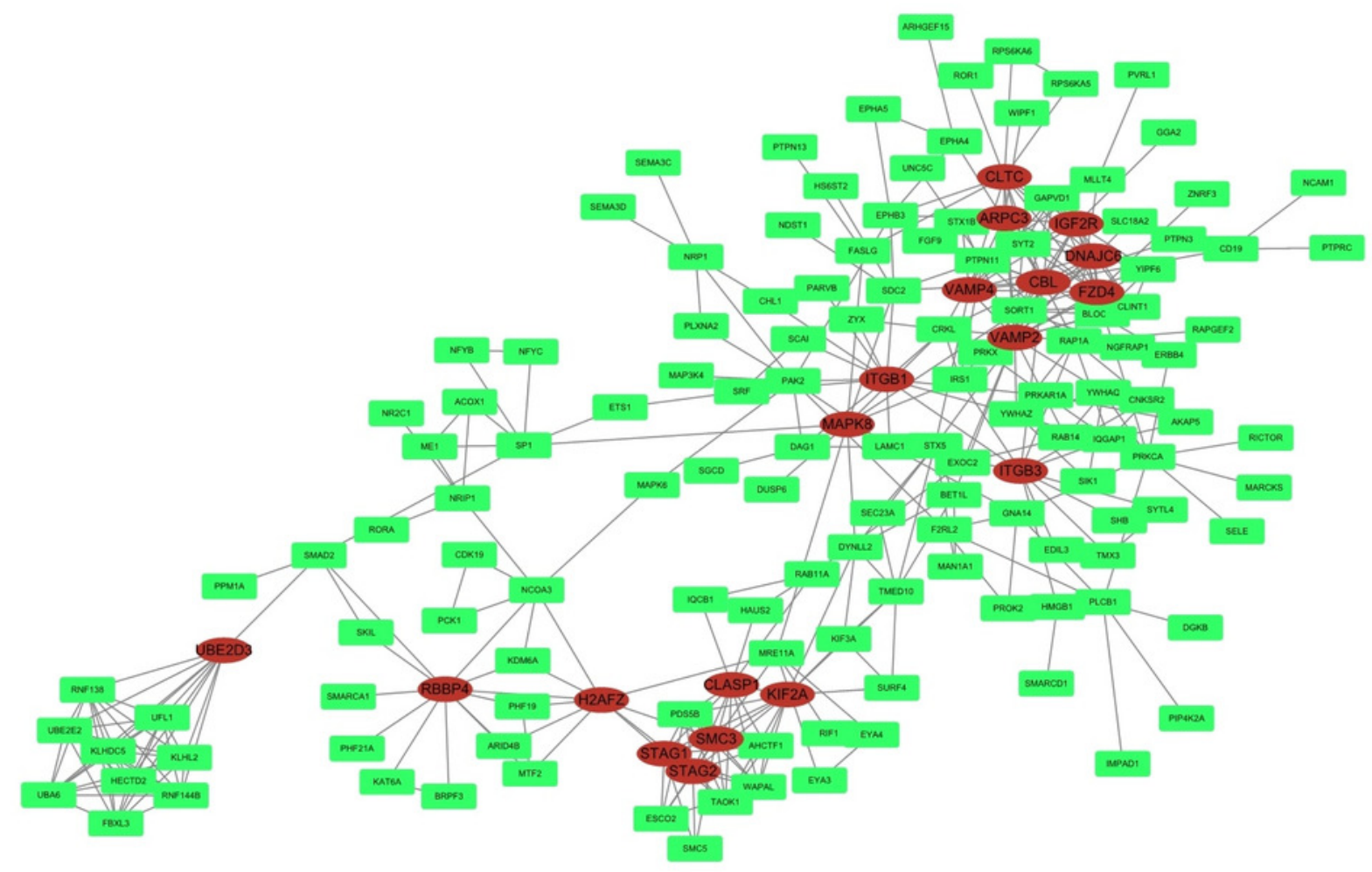


Table $\mathbf{1}$ (on next page)

Primers used in $\mathrm{qRT}-\mathrm{PCR}$ 
1 Table 1 primers used in qRT-PCR

\begin{tabular}{|c|c|c|c|}
\hline Gene & Forward primer $\left(5^{\prime}\right.$ to & Reverse primer $\left(5^{\prime}\right.$ to & Variation \\
\hline & $\left.3^{\prime}\right)$ & $\left.3^{\prime}\right)$ & tendency \\
\hline \multirow[t]{2}{*}{ Gapdh } & TGACTTCAACAGCGACA & CACCCTGTTGCTGTAGC & \\
\hline & CCCA & CAAA & \\
\hline hg38_circ_00136 & GGCAGACAGAAGGAAA & GCTTTTGCTCTTGGGTTC & down- \\
\hline 82 & CAGC & TG & regulated \\
\hline hg38_circ_00159 & CAAGGACTGCCTGATTG & GGAGGTGAGGGAGGA & down- \\
\hline 54 & ACAAG & GTTCA & regulated \\
\hline hsa_circ_001373 & TCCTTCCTGCCTTTAAACA & TGGTAGCACCCATTTGT & down- \\
\hline 8 & CAC & GAA & regulated \\
\hline hsa_circ_001322 & CCGGACACTTGTTTTCC & TTCTGTTTGTGAGCAAT & down- \\
\hline 5 & AGT & CATCC & regulated \\
\hline hg38_circ_00049 & CTGCCATAGGACAGGCT & GGCACAAAGACAGCCTA & up- \\
\hline 13 & GA & ATGA & regulated \\
\hline hsa_circ_000903 & TTAGGTGGTTGAGCGCC & GGGCAGTTCACCAACAGCTT & up- \\
\hline 5 & TGC & & regulated \\
\hline hsa_circ_000039 & ACAGAAGGGCAAGAGA & TTCCTTGGTCCTCGAGG & up- \\
\hline 2 & GGTGG & CAC & regulated \\
\hline hsa_circ_000401 & AGGGGAAGGATCTTAT & САCTGAGTCCATTCCCT & up- \\
\hline
\end{tabular}


2 


\section{Table 2 (on next page)}

The top five (ranked by $\mathrm{p}$-value) BP terms, CC terms, and MF terms 
1 Table 2. The top five(ranked by $\mathrm{p}$-value) BP terms, CC terms, and MF terms

\begin{tabular}{|c|c|c|c|}
\hline $\mathrm{GO}$ & $P$-value & Description & GO terms \\
\hline GO:0035335 & $1.20 \mathrm{E}-04$ & $\begin{array}{l}\text { peptidyl-tyrosine } \\
\text { dephosphorylation }\end{array}$ & biological_process \\
\hline GO:0030334 & $8.18 \mathrm{E}-04$ & regulation of cell migration & biological_process \\
\hline GO:0045944 & 0.0013274 & $\begin{array}{l}\text { positive regulation of transcription } \\
\text { from RNA polymerase II promoter }\end{array}$ & biological_process \\
\hline GO:0001525 & 0.0014863 & angiogenesis & biological_process \\
\hline GO:0002011 & 0.0018697 & $\begin{array}{l}\text { morphogenesis of an epithelial } \\
\text { sheet }\end{array}$ & biological_process \\
\hline GO:0005913 & 1.67E-04 & cell-cell adherens junction & cellular_component \\
\hline GO:0005654 & $2.11 \mathrm{E}-04$ & nucleoplasm & cellular_component \\
\hline GO:0005829 & 7.43E-04 & cytosol & cellular_component \\
\hline GO:0030027 & 7.79E-04 & lamellipodium & cellular_component \\
\hline GO:0005634 & $9.11 \mathrm{E}-04$ & nucleus & cellular_component \\
\hline GO:0005515 & $6.07 \mathrm{E}-10$ & protein binding & molecular_function \\
\hline GO:0004725 & $4.76 \mathrm{E}-04$ & $\begin{array}{l}\text { protein tyrosine phosphatase } \\
\text { activity }\end{array}$ & molecular_function \\
\hline GO:0098641 & 0.001694 & $\begin{array}{l}\text { cadherin binding involved in cell- } \\
\text { cell adhesion }\end{array}$ & molecular_function \\
\hline GO:0000978 & 0.003848 & $\begin{array}{l}\text { RNA polymerase II core promoter } \\
\text { proximal region sequence-specific } \\
\text { DNA binding }\end{array}$ & molecular_function \\
\hline GO:0001077 & 0.005654 & $\begin{array}{l}\text { transcriptional activator activity, } \\
\text { RNA polymerase II core promoter } \\
\text { proximal region sequence-specific } \\
\text { binding }\end{array}$ & molecular_function \\
\hline
\end{tabular}


Table 3(on next page)

The top five (ranked by gene count) KEGG pathways 
1 Figure 3. The top five(ranked by gene count) KEGG pathways

2

\begin{tabular}{|c|c|c|c|}
\hline Term & Count & PValue & Genes \\
\hline $\begin{array}{l}\text { MAPK signaling } \\
\text { pathway }\end{array}$ & 19 & $\begin{array}{r}0.00211 \\
4\end{array}$ & $\begin{array}{l}\text { PRKCA, FGF9, TAOK1, NLK, PPM1A, FASLG, PPM1B, } \\
\text { STK4, SRF, RPS6KA5, RPS6KA6, MAP3K4, CRKL, PAK2, } \\
\text { RASGRP3, RAP1A, MAPK8, RAPGEF2, DUSP6 }\end{array}$ \\
\hline Endocytosis & 15 & $\begin{array}{r}0.04983 \\
7\end{array}$ & $\begin{array}{l}\text { PARD6B, ERBB4, KIF5C, CBL, ASAP2, EEA1, SMAD2, PSD2, } \\
\text { CLTC, ARPC3, IGF2R, KIAA1033, DNAJC6, RAB11A, WIPF1 }\end{array}$ \\
\hline Axon guidance & 13 & 0.00105 & $\begin{array}{l}\text { EPHA5, ABLIM1, EPHA4, NRP1, PAK2, SEMA6D, PLXNA2, } \\
\text { ABLIM3, SEMA3D, SEMA3C, UNC5C, EPHB3, ITGB1 }\end{array}$ \\
\hline $\begin{array}{l}\text { Neurotrophin signaling } \\
\text { pathway }\end{array}$ & 9 & $\begin{array}{r}0.04726 \\
1\end{array}$ & $\begin{array}{l}\text { RPS6KA5, RPS6KA6, CRKL, RAP1A, SORT1， FASLG, } \\
\text { MAPK8, IRS1, PTPN11 }\end{array}$ \\
\hline $\begin{array}{l}\text { SNARE interactions in } \\
\text { vesicular transport }\end{array}$ & 5 & $\begin{array}{r}0.02571 \\
3\end{array}$ & STX5, VAMP4, BET1L, VAMP2, STX1B \\
\hline $\begin{array}{l}\text { Endocrine and other } \\
\text { factor-regulated } \\
\text { calcium reabsorption }\end{array}$ & 5 & 0.06235 & PRKCA, KL, RAB11A, CLTC, PLCB1 \\
\hline $\begin{array}{l}\text { Proximal tubule } \\
\text { bicarbonate } \\
\text { reclamation }\end{array}$ & 4 & $\begin{array}{r}0.03988 \\
7\end{array}$ & SLC38A3, GLS, GLUD1, PCK1 \\
\hline Circadian rhythm & 4 & $\begin{array}{r}0.08351 \\
4\end{array}$ & CRY2, BHLHE40, RORA, FBXL3 \\
\hline $\begin{array}{l}\text { African } \\
\text { trypanosomiasis }\end{array}$ & 4 & $\begin{array}{r}0.09665 \\
2\end{array}$ & PRKCA, FASLG, PLCB1, SELE \\
\hline
\end{tabular}

